\title{
Digital Imaging and Communication in Medicine- A Digital Window for Oral Pathology
}

\author{
Shubhangi Mhaske ${ }^{1}$, Ruhi Khan ${ }^{2}$, Monal Yuwanati ${ }^{3}$, Anish Gupta $^{4}$
}

\begin{abstract}
Recently, whole slide imaging technology allows glass slides to be scanned and viewed on a computer screen via dedicated software. This technology is referred as virtual microscopy and has created enormous opportunities in pathological training and education. Students are able to learn key histopathological skills, e.g. to identify areas of diagnostic relevance from an entire slide, via a web-based computer environment. Students no longer need to be in the same room as the slides. The Digital Imaging and Communications in Medicine (DICOM) Standard specifies a non-proprietary data interchange protocol, digital image format, and file structure for biomedical images and image-related information. The use of digitized histopathologic specimens (also known as whole-slide images (WSIs)) in clinical medicine requires compatibility with the Digital Imaging and Communications in Medicine (DICOM) standard. Knowledge of DICOM's benefits and realistic understanding of its limitations enable one to use the Standard effectively as the basis for a long term implementation strategy for image management and communications systems.
\end{abstract}

\section{Introduction}

The field of Pathology is undergoing a transformation in which digital imaging is becoming increasingly important. This transformation is fueled by the commercial availability of instruments for digitizing microscope slides.

The most widely used medical imaging standard is the Digital Imaging and Communications in Medicine $(\mathrm{DICOM})^{1}$, which is routinely used in several medical specialties, especially radiology. The Digital Imaging and Communications in Medicine (DICOM) Standard specifies a non-proprietary data interchange protocol, digital image format, and file structure for biomedical images and image-related information. DICOM addresses five general application areas:

(1) network image management, (2) network image interpretation management, (3) network print management, (4) imaging procedure management, (5) off-line storage media management. ${ }^{2}$ In pathology, digitization of whole microscope specimens has recently become possible with high-throughput slide scanners ${ }^{3}$. The digitized versions of microscope glass slides are called "virtual slides" or "whole-slide images" (WSIs). Acquiring, handling, and displaying WSIs is commonly called "virtual microscopy" (or whole-slide imaging) ${ }^{4,5}$ WSIs can be used for local viewing or, more practically, for remote viewing by transmitting them over networks.

Virtual slides have been around in a usable form since the early 1990s and, as a technology, are increasing in maturity. They have many innovative uses in education and training, and are becoming an essential tool in research practice. However, their use in routine diagnosis has yet to come. This review aims to give a basic introduction to virtual slides and application of DICOM in oral pathology

\section{What is a virtual slide?}

A virtual slide is a digital image produced by scanning a glass pathology slide at high resolution. Typically, virtual slide scanners use standard microscope lenses and light sources to obtain a microscopic image of the tissue. The resulting image is captured electronically with an image capture device similar to those seen in digital cameras (a so-called CCD or CMOS chip) to record all of the image data from the slide. The effective resolution of virtual slide scanners is similar to that of a microscope: of the order of $0.2-0.5 \mu \mathrm{m}$ per pixel (fig:1). Virtual slides differ from telepathology, although the end result may appear the same to the user. In telepathology, a microscope is remotely driven by the pathologist, and the image from the microscope is transmitted to them live. For virtual slides, however, the image acquisition and viewing need not happen at the same time. Virtual slides are only feasible due to the improvements in computer processing and storage capabilities of the last one to two decades; before these, the idea of scanning and transmitting gigapixel images would have been unthinkable. 


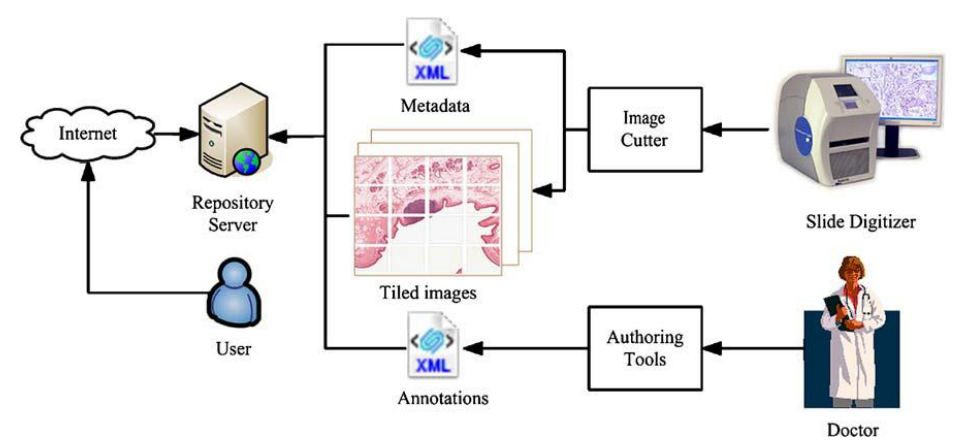

Figure1. Architecture of the virtual slide system.(Adopted from: Chung-Yueh Lien.et al, Journal of Digital Imaging, Vol 22, No 3 (June), 2009: pp 275-285)

\section{Producing a virtual slide}

Virtual slides differ from the image seen down the microscope in several ways due to the methods applied to acquire and store them. A summary of the important steps in producing virtual slides follows.

\section{Image focusing}

Histopathology and cytopathology specimens are three-dimensional (3D); virtual slides tend to be twodimensional (2D) images captured from the 3D object. This can be seen both as an advantage or disadvantage. On the one hand, a virtual slide can be seen as an optimally focussed 2D capture of a lumpy glass slide typically, virtual slide systems adjust the focus rapidly (tens or hundreds of times) during scanning to maintain a focused image in the final virtual slide. On the other hand, there may be important diagnostic information in the 3D slide that is lost by this approach. This is particularly true in cytopathology. Virtual slide vendors have addressed this by offering partial or whole slide 3D scanning - a significant drawback of this is that the already large digital image is made even larger when multiple scans in the third dimension (producing a so called zstack) are made.

\section{Image processing}

The image undergoes a variable degree of processing (alteration) during and after acquisition. For example, the image may be automatically sharpened (to enhance the definition of edges in the image) or the colour and brightness may be adjusted. This is standard practice in digital imaging (and can be used with conventional digital cameras to enhance photomicroscopy), but it is important to remember that the digital image is a processed digital reproduction of the captured image. ${ }^{6}$

\section{Digital Slides For Web-Based Education}

Appropriate software allows digital slides to be hosted on a centralized computer server and accessed by users anywhere in the world. The viewing software generally operates through a standard browser and allows the user to navigate the slide using on-screen controls. The user can 'drag and drop' the image on-screen to move around the slide or can move the image in discrete steps, left, right, up or down. An appropriate magnification can be selected from a discrete range or continuously changed by scrolling the mouse wheel to zoom in or out. The viewing software also provides a thumbnail overview of the entire slide, allowing users to maintain orientation and context, regardless of their magnification and position. Fig: $2^{7}$

\section{Viewing virtual slides}

Since microscope specimens are often up to $20 \times 30 \mathrm{~mm}$ in size, a WSI can contain up to $40 \mathrm{~GB}$ of uncompressed image data (with a scanning resolution $0.2-0.5 \mu \mathrm{m}$ per pixel) ${ }^{8}$. The amount of data increases further if scanning is done at a higher optical magnification and/or if several focus layers (along Z-axis) are scanned (e.g., in cytopathology) ${ }^{9}$. Due to the large size of WSIs, all viewing systems described to date apply the "on demand" principle: that is, only a user-requested area (with a desired resolution) of the WSI is decoded and displayed. Moreover, the large image size necessitates the use of lossy image compression.

Lossy compression can yield a 10- to 30-fold compression ratio compared to lossless compression, without affecting the diagnostic properties of a WSI ${ }^{10}$. Thus, a suitable image format for virtual microscopy needs to be based on an effective image compression algorithm, as well as to provide a sophisticated random access technique.

Virtual microscopy currently lacks a universally accepted WSI format. There are several proprietary image formats that are tied to specific scanner vendors, such as SVS (by Aperio Technologies, USA), NDP (by Hamamatsu Photonics, Japan), and Mirax (by Carl Zeiss MicroImaging, USA).It has previously shown that the 
open JPEG2000 standard is a suitable format for WSIs, allowing fast random slide access and efficient lossy compression ${ }^{11}$. Although JPEG2000 compression is computationally intensive, the process can be matched with current slide scanner speeds by utilizing multi-core processor environments. JPEG2000 is a family of standards supervised by the Joint Photographic Experts Group standardization committee $e^{12,13}$

The standard family currently consists of 13 parts, three of which are essential for virtual microscopy. Part 1 (Core Coding System) ${ }^{14}$ specifies the codestream syntax and the JP2 file format, which uses "jp2" as the common file extension. Part 2 (Extensions) ${ }^{15}$ provides extensions for the first part. Part 9 (Interactivity Tools, APIs, and Protocols) ${ }^{16}$ introduces the JPEG2000 Interactive Protocol (JPIP) for remote serving and viewing of JPEG2000 images.

There was previously developed and released a free JPEG2000 software package (called JVS, for JPEG2000 Virtual Slide) comprising WSI compression, viewing, and network server applications ${ }^{11}$. Della Mea et al. ${ }^{17}$ have presented a survey of currently available JPEG2000 viewing software.

Currently, the DICOM standard includes the basic parts of the JPEG2000 standard in Supplements $61^{18}$ and $105^{19}$. Supplement 106 (JPEG 2000 Interactive Protocol) ${ }^{20}$ describes two JPIP-based Transfer Syntaxes as methods of delivering image pixel data apart from patient data: the noncompressed JPIP Referenced Transfer Syntax and the Deflate-compressed ${ }^{21}$ JPIP Referenced Deflate Transfer Syntax.

When using the JPIP Transfer Syntaxes in a DICOM-based Picture Archiving and Communication System (PACS), a DICOM server sends its client a Uniform Resource Locator (URL) string that refers to the WSI pixel data provider (i.e., a JPIP server), together with the image name, which can be arbitrary and unrelated to patient data (as shown in Fig. 3). Upon receiving the pixel data provider reference, the client DICOM workstation can either use a built-in JPIP viewer or invoke an external one for retrieving the WSI fromthe specified JPIP server.

All network messaging between the PACS and the client end is done according to the DICOMprotocol, except the JPIP transmission, which is by default performed on top of the Hypertext Transfer Protocol $(\text { HTTP/1.1 })^{22}$ for compatibility with existing Web infrastructure, but it can also be done using a lowerlevel transport protocol (such as Transmission Control Protocol, TCP) ${ }^{23}$. Image serving performance of JPIP has been demonstrated to be excellent and upwards scalable in multi-client systems ${ }^{11,24}$.

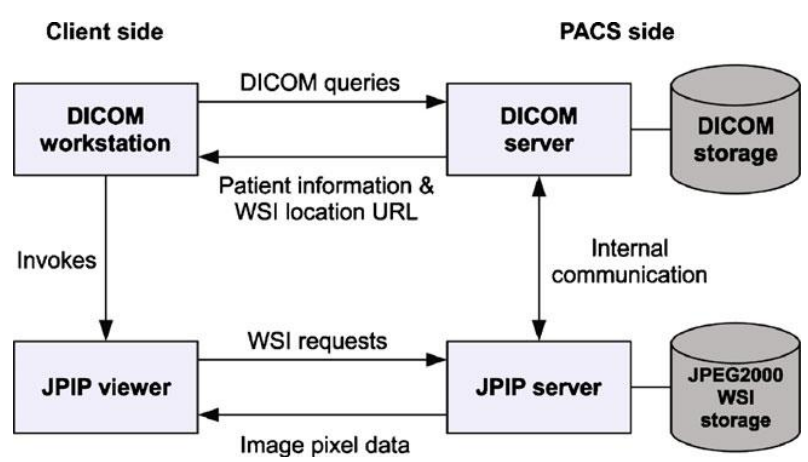

Fig 3. The principle of transmitting whole-slide images (WSIs) within a DICOM-based PACS by using JPEG2000 Interactive Protocol (JPIP). (Adopted from: Vilppu J. Tuominen and Jorma Isola. Journal of Digital Imaging, Vol 23, No 4 (August), 2010: pp 454-462) 


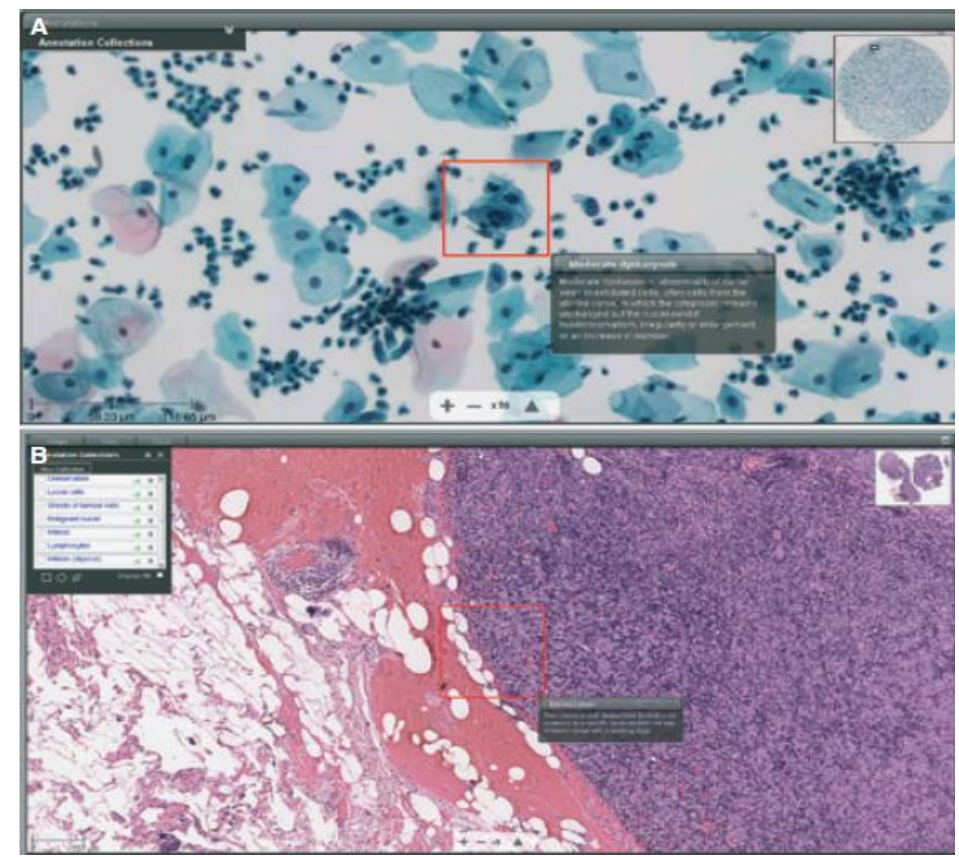

Fig.2. An example of annotations, showing how key features can be digitally marked and labeled on the slides. By clicking on an annotation label from the list (top left hand corner), the software relocates to that region of the slide, showing the important region of interest. Note also the thumbnail overview of the slide in the top right hand corner of the screen.( Adopted from: Peter W. Hamilton et al, APMIS 120:305-315)

\section{Advantages and disadvantages of virtual slides ${ }^{6}$}

The advantages and disadvantages of virtual slides are listed in Table 1.

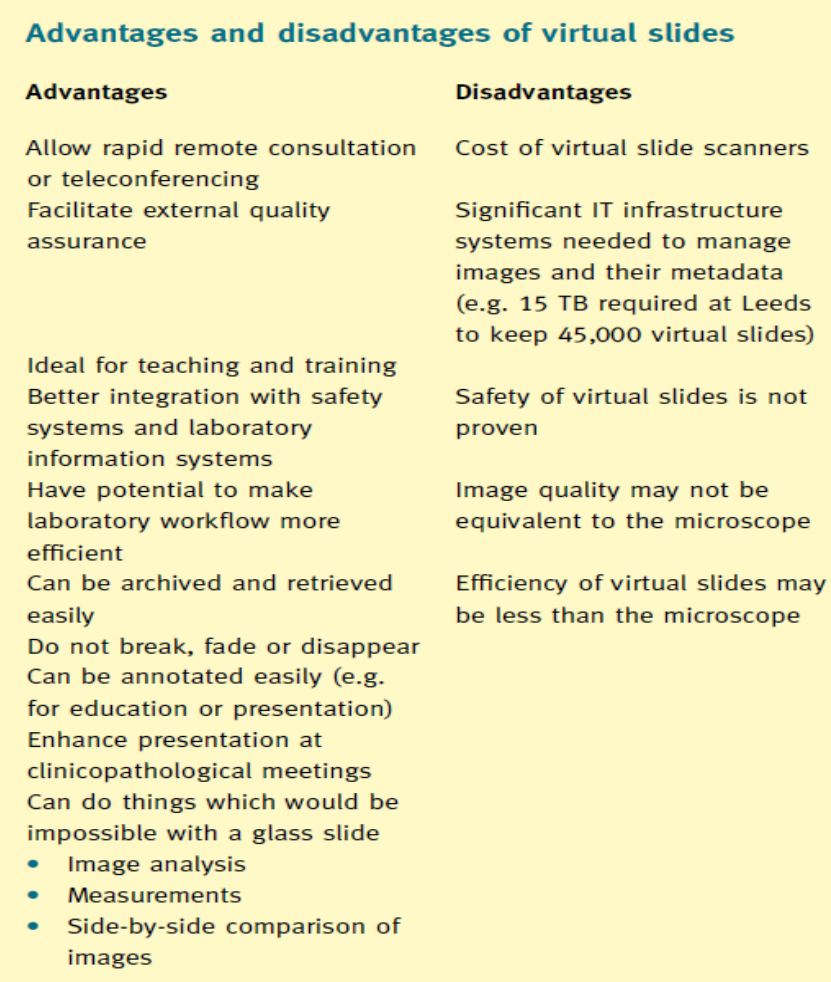

Table :1 


\section{Uses Of Virtual Slides In Education And Training \\ Undergraduate teaching}

With an increasing trend in medical schools away from microscope based tutorials, there is a risk that teaching of basic pathology will suffer. Several medical schools have developed e- learning based approaches to teaching microscopy - for example, see the excellent WebPath site. ${ }^{2}$ While this is undoubtedly of benefit, viewing static images does not offer the same interactivity as using a microscope. Virtual slides allow students to examine normal and diseased tissue in an interactive way, but without the complexity and logistic needs of a microscope laboratory. In addition, virtual slides can be integrated into e-learning material such as tutorials or online questionnaires, and can be annotated to guide students to areas of interest. In this context, virtual slides have already been successfully implemented in medical education at several institutions. ${ }^{3,4}$

\section{Postgraduate Training}

Familiarity with the microscope is not a problem in postgraduate training, but access to material is. During their training, pathologists are expected to combine reading with practical experience of interpreting slides. However, departmental and personal slide collections are often incomplete, can only be accessed by a small number of trainees at a time and may break, fade or be lost. Virtual slides offer the possibility of making large training resources of images available to trainees - slide libraries can offer thousands of validated images for trainees to study; again the material can be integrated into e-learning material. ${ }^{6}$

\section{Conclusions}

With the costs of scanners and computer storage dropping, and the enormous potential benefits of virtual slides, adoption of the technology will surely become more widespread. Impediments such as cost and the significant investment required in IT infrastructure (for storage, backup, and image transfer) will be balanced against the system performance improvements possible using virtual slides. However, there is still much work to be done before virtual slides become a safe and efficient alternative to the conventional microscope.

\section{References}

[1]. Klatt EC. Webpath: the Internet Pathology Laboratory for Medical Education. http://library.med.utah.edu/WebPath/webpath.html(accessed 1.07.08).

[2]. W. Dean bidgood, Steven C. Horii, Understanding and using dicom, the Data Interchange Standard for Biomedical Imaging. Journal of the American Medical Informatics Association Volume 4 Number 3 May / Jun 1997.

[3]. Krippendorf BB, Lough J. Complete and rapid switch from light microscopy to virtual microscopy for teaching medical histology.Anat Rec B New Anat 2005; 285: 19-25.

[4]. Kumar RK, Freeman B, Velan GM, De Permentier PJ. Integrating histology and histopathology teaching in practical classes using virtual slides. Anat Rec B New Anat 2006; 289: 128-33.

[5]. Furness P. A randomized controlled trial of the diagnostic accuracy of internet-based telepathology compared with conventionalmicroscopy. Histopathology 2007; 50: 266-73.

[6]. Darren Treanor Virtual slides: An Introduction, Diagnostic Histopathology 15:2

[7]. Peter w. Hamilton, Yinhai Wang Virtual microscopy and digital pathology in trainingand education. APMIS 120: 305-315

[8]. Rojo MG, García GB, Mateos CP, García JG, Vicente MC: Critical comparison of 31 commercially available digital slidesystems in pathology. Int J Surg Pathol 14(4):285-305, 2006.

[9]. Kalinski T, Zwönitzer R, Sel S, Evert M, Guenther T, Hofmann H, Bernarding J, Roessner A: Virtual 3D microscopyusing multiplane whole slide images in diagnostic pathology.Am J Clin Pathol 130(2):259-64, 2008.

[10]. Gao M, Bridgman P, Kumar S: Computer aided prostate cancer diagnosis using image enhancement and JPEG2000. Proc SPIE 5203:323-334, 2003

[11]. Tuominen VJ, Isola J: The application of JPEG2000 in virtual microscopy. J Digit Imaging, doi:10.1007/s10278-007-9090-z, 2007 Linking Whole-Slide Microscope Images With Dicom 461

[12]. Taubman DS, Marcellin MW: JPEG2000: Image Compression Fundamentals, Standards and Practice, Boston: Kluwer Academic, 2002

[13]. The Joint Photographic Experts Group Web site. Available at http://www.jpeg.org/. Accessed 19 October 2008

[14]. Information technology-JPEG 2000 image coding system: Core coding system. ISO/IEC 15444-1, 2000|ITU-T Recommendation T.800, 2002

[15]. Information technology-JPEG 2000 image coding system: Extensions. ISO/IEC 15444-2, 2002 | ITU-T Recommendation T.801, 2002

[16]. Information technology_JPEG 2000 image coding system: Interactivity tools, APIs and protocols. ISO/IEC 15444-9, 2005 | ITUT Recommendation T.808, 2005.

[17]. Della Mea V, Bortolotti N, Beltrami CA: A survey on non specialized off-the-shelf JPEG2000 viewers for digital microscopy use. Diagn Pathol 3(Suppl 1):S20, 2008

[18]. Digital Imaging and Communications in Medicine (DICOM), Supplement 61: JPEG 2000 Transfer Syntaxes. Available at $\mathrm{ftp} / / /$ medical.nema.org/medical/dicom/final/ sup61_ft. pdf. Accessed 9 October.

[19]. Digital Imaging and Communications in Medicine (DICOM), Supplement 105: JPEG 2000 Part 2 Multi-component Transfer Syntaxes. Available at ftp://medical.nema.org/ medical/dicom/final/sup105_ft.pdf. Accessed 9 October 2008

[20]. Digital Imaging and Communications in Medicine (DICOM), Supplement 106: JPEG 2000 Interactive Protocol. Available at ftp://medical.nema.org/medical/dicom/final/ sup106_ft.pdf. Accessed 9 October 2008 
[21]. The Internet Engineering Task Force (IETF), RFC 1951, DEFLATE Compressed Data Format Specification version 1.3. Available at http://www.ietf.org/rfc/rfc1951.txt. Accessed 9 October 2008

[22]. The Internet Engineering Task Force (IETF), RFC 2616, Hypertext Transfer Protocol -HTTP/1.1. Available at http:// www.ietf.org/rfc/rfc2616.txt. Accessed 9 October 2008

[23]. Taubman DS, Prandolini R: Architecture, philosophy and performance of JPIP: Internet protocol standard for JPEG2000. Proc SPIE 5150:791-805, 2003

[24]. Tian Y, Cai W, Sun J, Zhang J: A novel strategy to access high resolution DICOM medical images based on JPEG2000 interactive protocol. Proc SPIE 6919:691912, 2008 